\title{
Lung transplantation in children and young adults: a 20-year single-centre experience
}

\author{
Saskia Gruber*, Thomas Eiwegger*, Edith Nachbaur*, Kerstin Tiringer*, \\ Clemens Aigner", Peter Jaksch", Maya Keplinger", ", Walter Klepetko\#, \\ György Lang" ${ }^{\#}$ Shahrokh Taghavi" , Alexandra Graf', Irmgard Eichler*, \\ Thomas Frischer* and Zsolt Szépfalusi*
}

ABSTRACT: Lung transplantation in adults is an accepted therapeutic option, whereas there is ongoing debate on its positive impact on survival in children. We report our experience of the first 20 yrs of paediatric lung transplantation at a single centre in Austria.

Patient survival, organ survival and freedom from bronchiolitis obliterans were estimated by Kaplan-Meier curves. Pre- and post-transplant parameters were assessed and their influence on patient and organ survival evaluated by univariate tests and stepwise multivariate analyses.

A total of 55 transplantations were performed in $\mathbf{4 3}$ patients. 1- and 5-yr patient survival rates were $72.1 \%$ and $60.6 \%$, respectively, and $52.6 \%$ of patients were found to be free from bronchiolitis obliterans syndrome at 5 yrs post-transplant. Analysing different eras of transplantation suggests an improvement over the years with a 5 -yr survival rate of $70.6 \%$ in the second decade. A positive effect of pre-transplant diabetes mellitus and immunosuppression was found with the newer drug tacrolimus, and a negative effect of pre-transplant in-hospital admission was reported. A high rate of successful re-transplantation prolonged total patient survival.

KEYWORDS: Adolescents, children, cystic fibrosis, lung transplantation, single centre

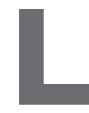

ung transplantation has emerged as an accepted therapy for end-stage lung disease in adult patients, whereas in the paediatric population it is still controversial [1-3]. Since the first successful lung transplant following the implementation of cyclosporin in 1983, the number of lung transplants performed worldwide has increased to approximately 2,500 per year [4]. Paediatric lung transplantation had scarcely been performed until the 1990s and even today represents only a minority of total procedures performed, accounting for up to approximately 80 procedures per year worldwide [5]. Therefore, transplantation in children is reserved to a very small number of centres, with only 30 to 40 institutions reporting paediatric lung transplantations [5]. The reasons are manifold, including the paucity of both donors and recipients, the investment required in developing paediatric lung transplant centres and scepticism about the outcome.

Survival after lung transplantation is still well below that of transplantation of other solid organs but has increased over the past decades [2, 5-7]. The subgroup of children and adolescents has been repeatedly reported to have poorer results $[3,6-8]$, leading to ongoing discussion on the beneficial effect of lung transplantation on survival in these patients $[2,3,6]$.

The Department for Thoracic Surgery at the Medical University of Vienna (Vienna, Austria) is one of the largest lung transplant centres in Europe, with the highest per head transplantation rate worldwide (14.1 per million inhabitants) [9]. The first lung transplantation in an adolescent cystic fibrosis (CF) patient treated at the Department of Paediatrics and Adolescent Medicine within the centre was performed in 1990. Since then our paediatric department has cared for 43 patients before and after lung transplantation.

This study describes the experience of the first 20 yrs of paediatric lung transplantation at a single centre in Austria. We assessed patient and graft survival and analysed which covariates had an effect on the outcome of the procedure.

Earn CME accreditation by answering questions about this article. You will find these at the back of the printed copy of this issue or online at www.erj.ersjournals.com/misc/cmeinfo.xhtml

AFFILIATIONS

*Dept of Pediatrics and Adolescent Medicine, Medical University of Vienna,

\#Dept of Thoracic Surgery, Medical University of Vienna,

"Dept of Anesthesia, General

Intensive Care and Pain Management, Medical University of Vienna, and

${ }^{+}$Dept of Medical Statistics, Medical University of Vienna, Vienna, Austria.

CORRESPONDENCE

T. Frischer

Dept of Pediatrics and Adolescent Medicine

Medical University of Vienna Waehringer Guertel 18-20

1090 Vienna

Austria

E-mail: thomas.frischer@

meduniwien.ac.at

Received:

May 312011

Accepted after revision:

Nov 292011

First published online:

Feb 232012 


\section{MATERIALS AND METHODS}

The study was approved by the local ethics committee and written consent was given by all participants in Austria.

\section{Patient collective}

The study population includes all lung transplanted patients that attended the Department of Paediatrics and Adolescent Medicine prior to transplantation, from the beginning of the procedure in April 1990 to January 2010. Most of the patients received their post-transplant care at our centre; however, some patients died while in the intensive care unit and others received part of their post-transplant care in their respective home country or at the local adult transplant ward. All transplantations were performed at the Department for Thoracic Surgery at the Medical University of Vienna.

Our patient cohort includes 12 young adults (aged $>17$ yrs at transplantation), which is due to the fact that all patients with $\mathrm{CF}$, disregarding age, were treated in the paediatric department until 1995

Due to the geographical location of Vienna, situated at the eastern border of Austria, our patient population includes a high proportion $(n=11)$ of foreign children and adolescents with a highly variable social and medical background. Since the first transplantation in an Italian child in 2003, a total of 11 patients from abroad, including Hungary, Italy, Czech Republic, Germany, Romania and Greece, have undergone transplantation at our centre. Selection and preparation for transplantation in these children was performed in conjunction with the respective home country. Post-transplant care was provided in Vienna in only four patients and as shared care in five patients. Two children received long-term follow-up at their local centre only. Adolescent patients from abroad transplanted by our surgery department but not presented at our paediatric ward before transplantation were not included in the study.

\section{Investigated parameters}

We assessed various pre-and post-transplant parameters and included these data in a detailed patient registry. Pre-transplant parameters were patient age, sex, body mass index (BMI), underlying disease, nationality, lung function, bacterial infections, cytomegalovirus (CMV) status, preformed antibodies and diabetes mellitus, as well as invasive ventilation and in-hospital admission at time of transplantation. Perioperative parameters included procedure of choice for transplantation, implementation of extracorporal membrane oxygenation (ECMO), ischaemia time, crossmatch, immunosuppression and induction therapy. Post-operative factors were time on intensive care unit, total in-hospital time post-transplant, and occurrence of bronchiolitis obliterans syndrome (BOS) and other post-transplant complications (kidney function impairment, lymphoproliferative disease and neurological complications), as well as causes of graft failure and death.

\section{Statistical analysis}

Kaplan-Meier curves were calculated to estimate being freedom from BOS, patient survival and organ survival. Tests on all pre- and peritransplant parameters (all measured within 4 months before transplantation) were performed to identify risk factors for post-transplant survival and BOS occurrence.
This was performed by univariate Cox regression models for the first transplant for each of the end-points: patient survival, transplant survival and freedom from BOS. Finally, a multiple extended Cox regression model was calculated for each endpoint accounting for the parameters being significant in the univariate analysis $(\mathrm{p}<0.05)$. All analyses were performed using SPSS version 17.0 (SPSS Inc., Chicago, IL, USA) or R 2.9.2 (www.r-project.org).

\section{Transplant procedure}

Surgical approach was uni- or bilateral anterolateral thoracotomy or bilateral thoracotomy with transverse sternotomy (clamshell technique). Since 2001, intra-operative ECMO has been applied in the majority of patients $(69.8 \%)$ as reported by Aigner et al. [10]. Mean ischaemia time (second lung in double lung transplantation) was $335.3 \pm 72.7 \mathrm{~min}$.

Immunosuppression at time of transplantation consisted of different combinations of corticosteroids (100\%), antiproliferative agents (azathioprine 18.6\% and mycophenolate mofetil $79.1 \%$ ) and calcineurin inhibitors (cyclosporin A $46.5 \%$ and tacrolimus $53.5 \%$ ). In 15 patients, immunosuppression was switched during the post-operative course. Of these, five $(11.4 \%)$ received rapamycin at some time-point post-transplantation. Induction was performed in the majority of patients, using antithymocyte globulin in $54.8 \%$ and anti-interleukin-2 receptor antibodies in $9.5 \%$. One patient received anti-CD3 antibodies after re-transplantation.

CMV prophylaxis was performed with CMV hyperimmune globulin once a week for 4 weeks post-transplant and ganciclovir for 3 months. Since 2006, ganciclovir prophylaxis has been given throughout the first post-transplant year in high-risk patients (CMV-positive donor, CMV-negative recipient).

\section{RESULTS}

43 patients (22 male, 21 female) undergoing a total of 55 transplantations (31 double lung, six single lung, eight bilateral lobar, two unilateral lobar, seven split lung and one heart-lung transplantation) were included in the present study. Two living donor transplantations were performed. At the time of analysis, 21 patients had died (three of them after re-transplantation), eight were re-transplanted and two re-re-transplanted.

Mean \pm SD age at first transplantation was $15.0 \pm 7.1 \mathrm{yrs}$, ranging from 6 months to 30.4 yrs. $31(72.7 \%)$ patients were aged $<18$ yrs and $10(23.3 \%)$ were aged $<10$ yrs. The most common cause for transplantation was CF (56.4\%), followed by BOS after first transplantation (21.8\%). Indications for transplantation are presented in table 1 . Re-transplantation was performed after a median of 32 months (range 2 days to 144 months).

Almost half of patients (40\%) needed in-hospital care at time of transplantation and $25 \%$ were on invasive ventilation. Five individuals were bridged to transplantation with ECMO. A detailed list of pre- and post-operative factors of all transplantations is given in table 2. Patient characteristics according to differences eras of transplantation are presented in table 3.

30-day transplant survival was $89.1 \%$; causes of early death or graft failure were multi-organ failure, primary transplant failure, intracerebral haemorrhage, intracerebral infarction and acute rejection. The most common causes of death during 


\begin{tabular}{|c|c|c|}
\hline TABLE 1 & \multicolumn{2}{|c|}{$\begin{array}{l}\text { Indications for transplantation of all } 55 \\
\text { transplantations }\end{array}$} \\
\hline \multicolumn{2}{|c|}{ Cystic fibrosis } & $31(56.4)$ \\
\hline \multicolumn{2}{|c|}{ Primary pulmonary hypertension } & $4(7.3)$ \\
\hline \multicolumn{2}{|c|}{ Graft versus host disease after BMT } & $2(3.6)$ \\
\hline \multicolumn{2}{|c|}{ Bronchopulmonary dysplasia } & $1(1.8)$ \\
\hline \multicolumn{2}{|c|}{ Cystic adenoid malformation } & $1(1.8)$ \\
\hline \multicolumn{2}{|c|}{ Idiopathic pulmonary haemosiderosis } & $1(1.8)$ \\
\hline \multicolumn{2}{|c|}{ Idiopathic lung fibrosis } & $1(1.8)$ \\
\hline \multicolumn{2}{|c|}{ Congenital alveolarproteinosis* } & $1(1.8)$ \\
\hline \multicolumn{2}{|c|}{ Cardiac malformation with SPH } & $1(1.8)$ \\
\hline \multicolumn{3}{|c|}{ Re-transplantation } \\
\hline \multicolumn{2}{|c|}{ BOS } & $7(12.7)$ \\
\hline \multicolumn{2}{|c|}{ Untreatable acute rejection } & $3(5.5)$ \\
\hline \multicolumn{2}{|c|}{ Insufficient lung function after first transplantation } & $2(3.6)$ \\
\hline
\end{tabular}

Data are presented as $n(\%)$. BMT: bone marrow transplantation; SPH: secondary pulmonary hypertension; BOS: bronchiolitis obliterans. \#: ABCA3 mutation

total follow-up were infections and BOS (both 28.6\%). Other causes were intracerebral haemorrhage/infarction and acute rejection (both 9.5\%), as well as cardiac complications, pulmonary bleeding, primary organ failure, carcinoma and multi-organ failure in one patient each $(23.3 \%)$. We did not observe recurrence of underlying disease, despite the fact that our cohort includes eight patients suffering from (possibly) immunologically mediated diseases (idiopathic lung fibrosis, idiopathic pulmonary haemosiderosis, graft versus host disease and primary pulmonary hypertension).

5 yrs post-transplant, kidney function was impaired in $66.7 \%$ of patients (mild $46.7 \%$ and moderate $76.7 \%$ ), with nine $(20.1 \%$ ) patients requiring dialysis at some point during transplant follow-up. 14 (32.6\%) patients showed neurological complications, including cerebral embolism, cerebral bleeding, posterior reversible encephalopathy syndrome and epileptic seizures, while no post-transplant proliferative lymphoproliferative disease occurred during total follow-up period. One patient developed adenocarcinoma of the lung.

Median estimated survival (Kaplan-Meier) of all 43 patients was 112 months (95\% CI 65.6-158.4), ranging from 2 days to 201 months (fig. 1a). 1-, 5- and 10-yr patient survival rates after transplantation were $72.1,60.6$ and $38.9 \%$, respectively.

Median estimated transplant survival of all 55 transplantations was 71 months (95\% CI 26.2-115.8), ranging from 2 days to 158 months (fig. 1b). 1-, 5- and 10-yr graft survival rates were $70.9,51.2$ and $15 \%$, respectively. Graft survival of re-transplantation (median survival 76 months; 95\% CI 22.2-115.7) was comparable to that of primary transplantation (median survival 71 months; 95\% CI 0-206.6).

Freedom from BOS was reported in 96.3 and $53.8 \%$ of patients at 1 - and 5-yrs post-transplant, respectively. Median time to occurrence of BOS was 48 months (95\% CI 29.8-66.2). Median transplant survival after occurrence of BOS was 31 months (95\% CI 13.9-48.1).

\section{TABLE 2 Characteristics of all 43 patients at first transplantation}

\begin{tabular}{|c|c|}
\hline Male/female & $51.2 / 48.8$ \\
\hline Age at transplantation yrs & $15.0(0.5-30.4)$ \\
\hline BMI & $15.5(11.0-25)$ \\
\hline \multicolumn{2}{|l|}{ Lung function } \\
\hline FVC \% pred & $37.6(17.0-93.3)$ \\
\hline FEV $1 \%$ pred & $23.9(14.0-107.6$ \\
\hline MEF50 \% pred & $10.6(2.8-97.3)$ \\
\hline Shared care & 25.6 \\
\hline Waiting time days & $66.5(1-339)$ \\
\hline Preformed antibodies & 36.4 \\
\hline Positive crossmatch & 33.3 \\
\hline High CMV risk & 31.0 \\
\hline Diabetes mellitus & 25.6 \\
\hline Invasive ventilation before transplantation & 25 \\
\hline In-hospital admission before transplantation & 40 \\
\hline \multicolumn{2}{|l|}{ Bacterial colonisation } \\
\hline Staphylococcus aureus & 27.9 \\
\hline Pseudomonas aeruginosa & 67.4 \\
\hline Burkholderia cepacia & 7.0 \\
\hline \multicolumn{2}{|l|}{ Type of transplantation } \\
\hline Double lung & 69.8 \\
\hline Bilateral lobar & 16.3 \\
\hline Unilateral lobar & 2.3 \\
\hline Split lung & 9.3 \\
\hline Heart-lung & 2.3 \\
\hline Operation on ECMO & 73.2 \\
\hline Ischaemia time ${ }^{\#} \min$ & $337.3(172-555)$ \\
\hline 30-day transplant survival & 89.1 \\
\hline Post-operative days on ICU & $22.4(3-100)$ \\
\hline Post-operative days in hospital & $43.7(3-100)$ \\
\hline \multicolumn{2}{|l|}{ Induction therapy } \\
\hline None & 35.7 \\
\hline ATG & 54.8 \\
\hline IL-2R antagonists & 9.5 \\
\hline
\end{tabular}

Immunosuppression

CsA/tacrolimus

$46.5 / 53.5$

Azathioprine/MMF

$19.0 / 81.0$

Cause of graft failure/death

Infection

28.6

BOS

28.6

Acute rejection $\quad 9.6$

Intracerebral bleeding/infarction $\quad 9.6$

Other" 23.8

Data are presented as \% or mean (range), lung function and waiting time are presented as median (range). BMl: body mass index; FVC: forced vital capacity; \% pred: \% predicted; FEV1: forced expiratory volume in $1 \mathrm{~s}$; MEF50: mean maximal expiratory flow; CMV: cytomegalovirus; ECMO: extracorporeal membrane oxygenation; ICU: intensive care unit; ATG: antithymocyte globulins; IL-2R: interleukin-2 receptor; CsA: cyclosporine A; MMF: mycophenolate

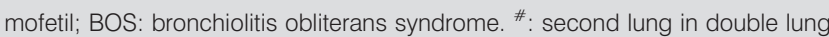
transplantation; ": acute rejection, primary organ failure, multi-organ failure, cardiac complications, intracerebral haemorrhage or pulmonary bleeding. 
TABLE 3 Patient characteristics according to different eras of transplantation

\begin{tabular}{|c|c|c|c|}
\hline & 1990-1999 & 2000-2009 & $\mathrm{p}$-value \\
\hline Subjects $\mathbf{n}$ & 18 & 37 & \\
\hline Age at transplantation yrs & $18.3(4.3-30.4)$ & $13.0(0.5-21.2)$ & 0.027 \\
\hline BMI & $14.9(12.3-17.4)$ & $15.8(11.0-25)$ & NS \\
\hline Shared care & 18.8 & 29.6 & NS \\
\hline FEV $1 \%$ pred & $20.5(14.0-31.0)$ & $27.6(15.7-107.6)$ & 0.031 \\
\hline MEF50 \% pred & $9.0(2.8-26.3)$ & $11.4(4.3-97.3)$ & NS \\
\hline Underlying disease CF & 93.8 & 59.3 & 0.017 \\
\hline Diabetes mellitus & 37.5 & 18.5 & NS \\
\hline Ischaemia time* min & $299.4(172-360)$ & $358.3(280-555)$ & NS \\
\hline In-hospital admission before transplantation & 40.0 & 40.0 & NS \\
\hline Waiting time days & $57(1-199)$ & $88(1-339)$ & NS \\
\hline \multicolumn{4}{|l|}{ Bacterial colonisation } \\
\hline Staphylococcus aureus & 31.3 & 25.9 & NS \\
\hline Pseudomonas aeruginosa & 87.5 & 55.6 & 0.031 \\
\hline Burkholderia cepacia & 12.5 & 3.7 & NS \\
\hline Transplantation on ЕСMO & 35.7 & 92.6 & $<0.001$ \\
\hline \multicolumn{4}{|l|}{ Induction therapy } \\
\hline ATG & 75.0 & 42.3 & NS \\
\hline IL-2R antagonist & 6.3 & 11.5 & NS \\
\hline \multicolumn{4}{|l|}{ Immunosuppression } \\
\hline
\end{tabular}

Data are presented as \% or mean (range), unless otherwise stated; lung function and waiting time are presented as median (range). BMI: body mass index; FVC: forced vital capacity; \% pred: \% predicted; FEV1: forced expiratory volume in $1 \mathrm{~s}$; MEF50: mean maximal expiratory flow; CF: cystic fibrosis; CMV: cytomegalovirus; ECMO: extracorporeal membrane oxygenation; ATG: antithymocyte globulins; IL-2R: interleukin 2 receptor; CsA: cyclosporine A; MMF: mycophenolate mofetil; NS: nonsignificant. *: second lung in double lung transplantation.

In 31 patients aged $<18$ yrs at first transplantation, outcome was comparable to that of the total sample. Median patient survival was 93 months and median organ survival was 39 months (95\% CI 0-88.4) (fig. 2a). 1- and 5-yr patient survival rates were 71 and $57.1 \%$, respectively, and 1- and 5-yr organ survival rates 71 and $42.9 \%$, respectively. Freedom from BOS was observed in 94.1 and $52.6 \% 1$ - and 5-yrs post-transplant, respectively. The median time to BOS was 40 months $(95 \% \mathrm{CI}$ 30.4-49.6).

When comparing the outcome according to different eras of the observation period (1990-1999 versus 2000-2009), there was a trend for better results in the second decade (fig. 2b). Median transplant survival increased from 37 months (95\% CI 0-89.9) to 75 months (95\% CI 25.5-124.5). 1-yr organ survival increased from 62.7 to $77.8 \%$ and 5 -yr survival from 43.8 to $52.9 \%$. The same trend was found regarding patient survival (1-yr survival: 62.7 to $77.8 \%$; 5-yr survival: 50 to $70.6 \%$ ). However, these differences were not statistically significant. Freddom from BOS at 5-yrs post-transplant increased significantly from 14.3 to $75 \%$ $(\mathrm{p}=0.019)$. Time to occurrence of BOS increased from a median of 39 to 81 months (nonsignificant).

Pre-transplant diabetes mellitus was associated with a significantly better patient $(p=0.007)$ but not organ survival, as well as with increased 5 -yr patient survival rates (47.8 versus $90.0 \%, \mathrm{p}=0.023$ ) (fig. 3a). This effect persisted in multivariate analysis.

A better survival of patients was observed in those receiving the newer immunosuppressive drug tacrolimus compared to patients treated with cyclosporine A (CsA) in uni- and multivariate analysis. This was reported for median patient $(p<0.03)$ (fig. $3 b$ ) and organ survival (borderline, $p=0.049$ ), as well as for 5 -yr patient $(\mathrm{p}<0.01)$ and organ survival rates (borderline, $\mathrm{p}=$ 0.056). We also observed a nonsignificant advantage of mycophenolate mofetil over azathioprine in patient (37 versus 158 months) and organ survival rates (12 versus 72 months). Both CsA and mycophenolate mofetil have been used more frequently in recent years; thus, other non-assessed time-dependent parameters might interact with the results. 

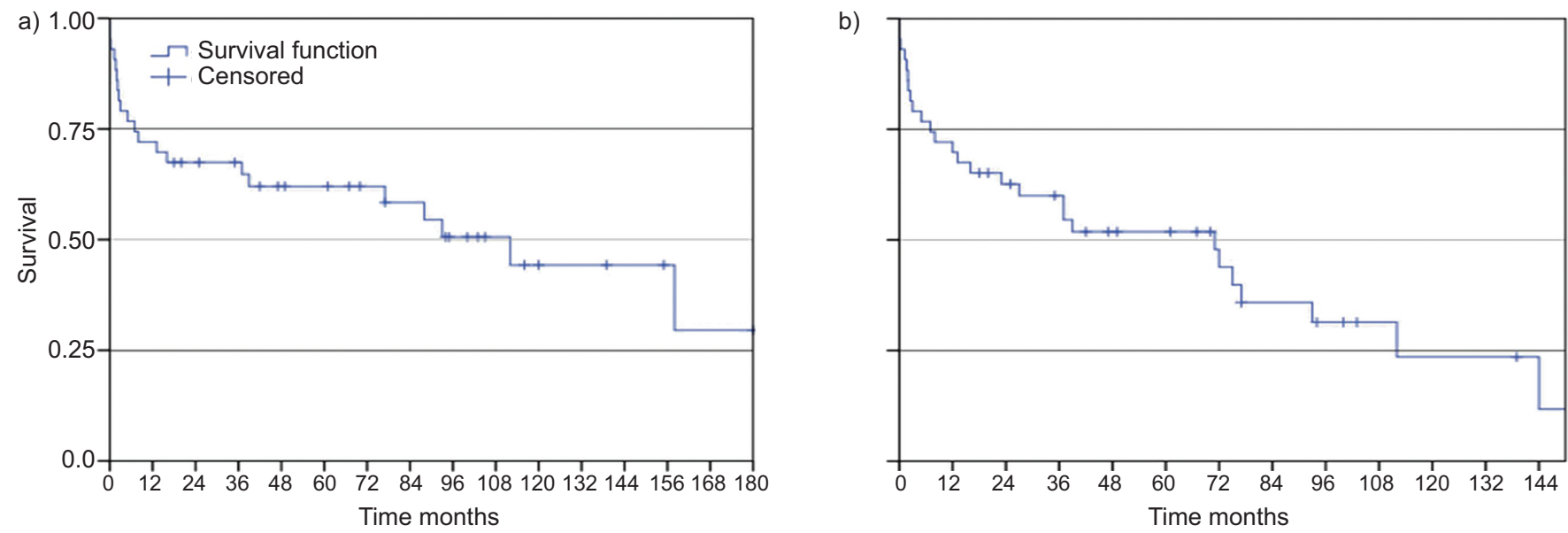

FIGURE 1. a) Patient survival ( $\mathrm{n}=43$ ). Median survival was 112 months, and 1-, 5 - and 10-yr patient survival was $72.1,60.6$ and $38.9 \%$, respectively. b) Transplant survival $(n=55)$. Median survival was 71 months, and 1-, 5- and 10-yr organ survival was 70.9, 51.2 and $15.0 \%$, respectively.

Moreover, in-hospital admission at time of transplantation was a significant parameter in univariate analysis. Patients requiring in-hospital care $(40 \%)$ had worse 1-yr transplant survival than patients who were well enough to be treated at home $(p=0.009)$. This effect was not present for 5-yr survival, which was similar in both groups. A similar trend was found for patients on invasive ventilation $(25 \%)$ before transplantation (1-yr transplant survival 50 versus $76 \%$ ), but this showed only borderline significance $(\mathrm{p}=0.06)$.

We found no statistically significant effect on post-transplant occurrence of BOS, patient and organ survival for the parameters patient age, underlying disease (CF versus non-CF), nationality, type of transplantation, lung function (forced vital capacity, forced expiratory volume in $1 \mathrm{~s}$ and mean maximal expiratory flow), BMI, bacterial colonisation (Burkholderia cepacia, Staphylococcus aureus and Pseudomonas aeruginosa), time on waiting list, crossmatch, presence of preformed antibodies, transplantation on ECMO, induction therapy, CMV status or intraoperative ischaemia time.

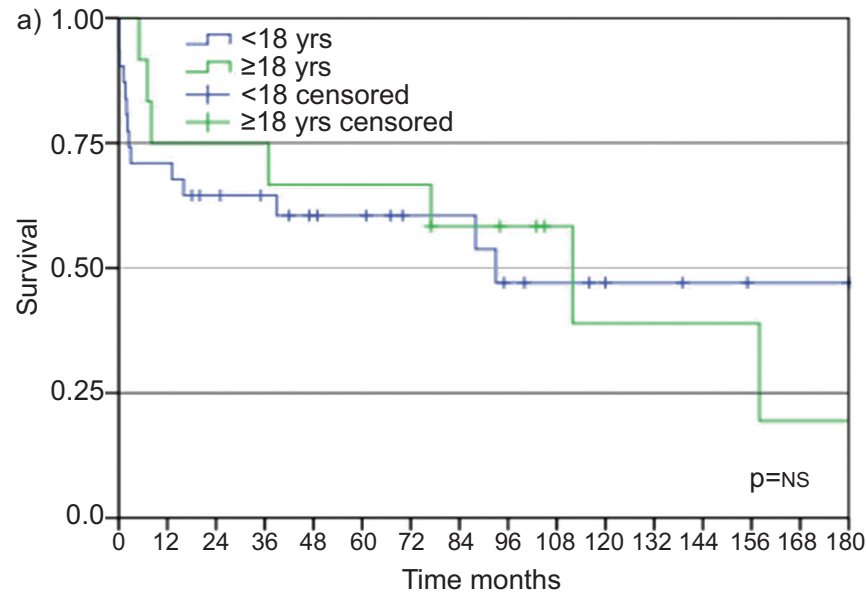

In multivariate analysis, both diabetes and immunosuppression with tacrolimus were found to have a positive influence on patient survival $(p=0.033)$, whereas for organ survival and freedom from BOS no independent risk factor was identified.

\section{DISCUSSION}

Analysing the first 20 yrs of lung transplantation in children, adolescents and young adults at our centre, we observed a median estimated total patient survival of 112 months. 1-, 5and 10-yr patient survival rates after transplantation were 72.1, 60.6 and $38.9 \%$, respectively. Stratification of data according to different eras of transplantation (1990-1999 versus 2000-2009) points toward an improvement of transplant and organ survival, as well as decreased occurrence of BOS over the years, with a 5-yr survival rate in the second decade of $70.6 \%$. Moreover, pre-transplant diabetes mellitus and the newer immunosuppressant tacrolimus have been shown to be associated with an improved survival, whereas pre-transplant in-hospital admission was associated with decreased early survival in univariate analysis.

b)

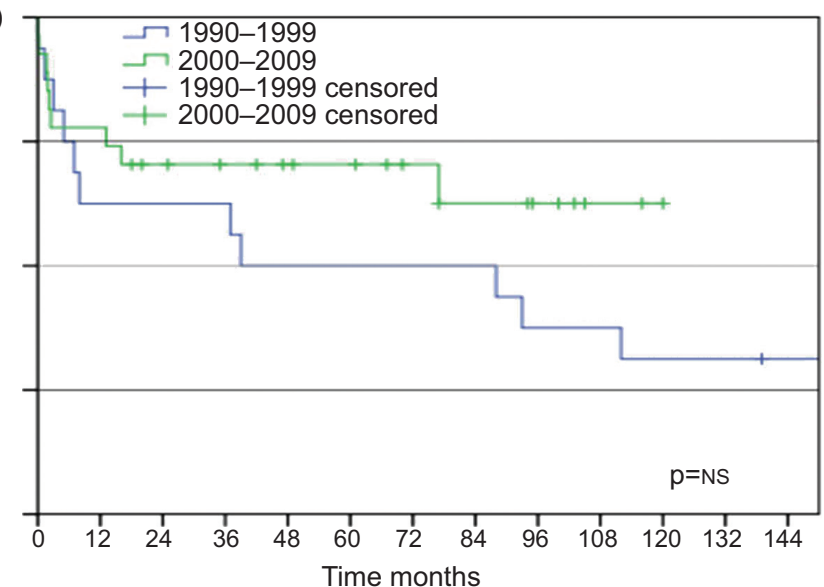

FIGURE 2. Patient survival according to a) age at first transplantation and b) transplant era (1990-1999 versus 2000-2009). a) In children aged 6 months to 17 yrs median survival was 93 months, and 1- and 5-yr survival were 71 and 57.1\%, respectively. In adults aged 18-30 yrs median survival was 112 months, and 1- and 5-yr survival were 75 and 66.7\%, respectively. b) In 1990-1999, median survival was 39 months, and 1- and 5-yr survival were 62.7 and 50.0\%, respectively. In 2000-2009, median survival was not available (due to small number of events), and 1 - and 5 -yr survival were 77.8 and $70.6 \%$, respectively. Ns: nonsignificant. 

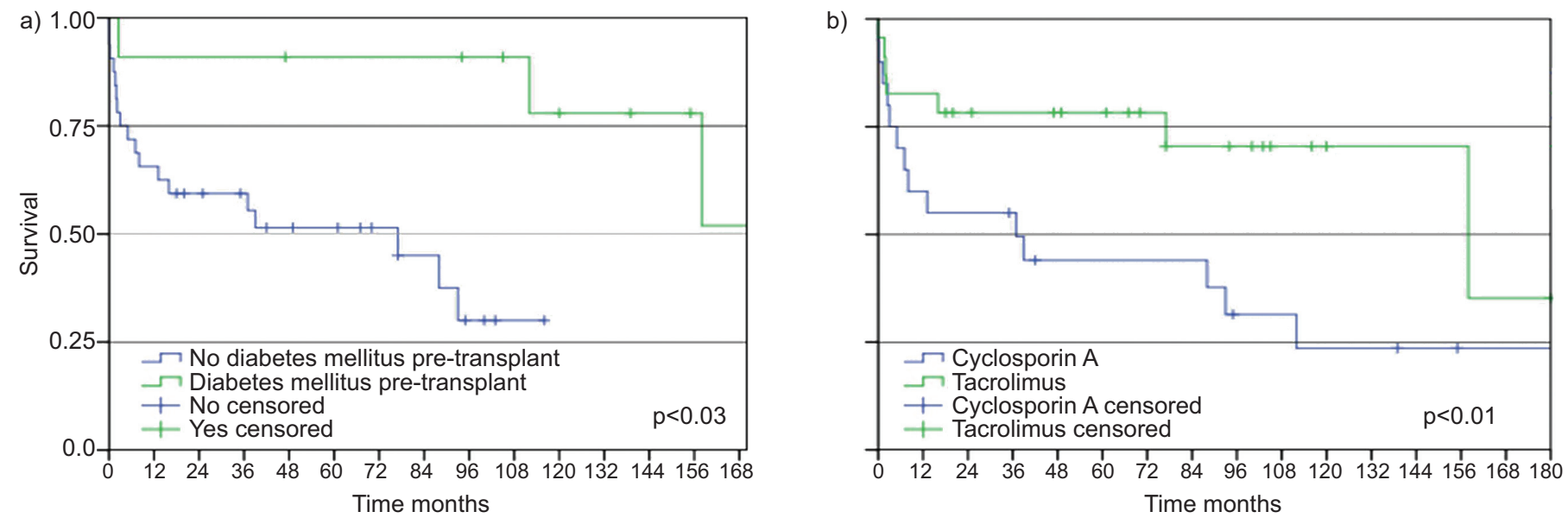

FIGURE 3. Patient survival according to a) pre-transplant diabetes mellitus and b) calcineurin inhibitor (cycloporin A versus tacrolimus). a) Pre-transplant diabetes mellitus median survival was not available (small number of events), and 1- and 5-yr survival were $90.9 \%$ and $90.0 \%$, respectively. No pre-transplant diabetes mellitus median survival was 77 months, and 1- and 5-yr survival were 65.6 and 47.8\%, respectively. b) Cyclosporin A median survival was 39 months, and 1- and 5-yr survival were 62.7 and $50.0 \%$, respectively. Tacrolimus median survival was not available (due to small number of events), and 1- and 5-yr survival were 77.8 and $70.6 \%$, respectively.

Our results exceed the worldwide results presented by the International Society of Heart and Lung Transplantation (ISHLT) registry in 2010 (5 yr survival: 1990-2008, 48\%; 2002$2008,52 \%$ ) [5]. Analysing the data of large single centres we have shown comparable results: Hannover, 2009: 1-yr survival 69\%, 5-yr survival 44\% [7]; Great Ormond Street Hospital (London, UK), 2004: 5-yr survival in 1994 27\%, more recent era 57\% [11]; St. Louis Children's Hospital (St Louis, MO, USA), 1990-2002: 1-yr survival 77\%, 5-yr survival 54\% [12]; and Zurich, 1992-2007 children and adults: 1-yr survival 86\%, 5-yr survival $68 \%$ [2]. Likewise, improvement over the years has been demonstrated in other reports of paediatric and adult lung transplantation $[2,5,11]$. However, comparison of our results to other lung transplant centres is difficult due to various special characteristics of our sample.

First, we have an increasing amount of patients from abroad, with a varying degree of shared care before and after transplantation. This is challenging in many ways. On-site pre-transplant evaluation, especially regarding psychosocial aspects, is limited in time and quality due to language barriers. Language barriers also complicate early post-transplant care that takes place at our transplant centre. Long-term follow-up is performed as shared or local care in the patient's home country; in this period most problems arise from limited access to experienced transplant units, communication problems between the different healthcare centres and financial issues. We show a similar short-term survival of foreign patients compared to patients residing in Austria; however, long-term data are still missing and often difficult to obtain.

Secondly, we have a high rate of critically ill children, as demonstrated by the high proportion of patients that had to be treated in hospital $(40 \%)$, were on invasive ventilation $(25 \%)$ or were on ECMO (12.4\%) before transplantation (table 2). This might account for the rather high mortality in the first posttransplant year in our cohort. In fact, we showed a statistically significant decreased 1-yr survival in patients that were admitted to hospital before transplantation ( $\mathrm{p}<0.01)$, as well as in patients on invasive ventilation (borderline significance).
This is in line with other reports describing pre-transplant mechanical ventilation to be a significant risk factor for morbidity and mortality in adults and children $[5,13,14]$.

Thirdly, we have a high proportion of patients that received reor re-re-transplantation $(23.3 \%)$. Re-transplantation remains the only therapeutic option in some cases of severe primary graft dysfunction, severe untreatable acute rejection and advanced BOS. However, due to the overall scarcity of donor organs and uncertain outcome its value has been questioned. One explanation for our high re-transplant rate might be the donor legislation in Austria (presumed consent system), leading to a comparable good organ supply [15]. Overall, numbers of paediatric re-transplantations are low, with only 74 paediatric transplantations performed worldwide since 1994 [5]. Re-transplantation has repeatedly been reported to have a poorer survival compared to primary transplantation in children and adults [16-20]. At our centre, re-transplantation has previously been shown to have good survival rates in selected adult patients [21]. In addition, in paediatric recipients in the present study, we report survival rates after re-transplantation that are comparable to those after primary transplantation. This leads to an increased overall patient survival, resulting in very good longterm survival rates.

Finally, our sample involves some young adults transplanted in the first years of the programme as a result of the lack of care centres for adult CF patients at that time. As we planned the study as a single centre analysis, we decided to include these patients into the general analysis. The outcome did not differ significantly between children and young adults.

We report a trend for a better outcome of patients transplanted in the second decade. However, while occurrence of BOS at 5yr post-transplant was significantly lower after 2000, neither increase in patient survival nor in transplant survival showed statistical significance. Several factors might contribute to these results, including the multiple time-dependent factors (induction therapy, immunosuppressive and anti-microbial therapy, transplant technique, intensive care and experience of involved 
care takers) changing over the follow-up period. In addition, small patient numbers may limit statistical analysis. In the annual registry of ISHLT, which includes data from all paediatric transplantations performed worldwide, significantly improved survival rates were demonstrated only for the last few years [5]. In our cohort, patients transplanted in the first and second era of the transplant programme (table 3) differed in age, underlying disease, proportion of transplantation on ECMO, immunosuppressive therapy, forced expiratory volume in $1 \mathrm{~s}$ and Pseudomonas colonisation. Some of these factors, such as newer immunosuppressive drugs, might have a positive impact on outcome, whereas others, such as younger patient age, might impair survival.

Interestingly, we found a significantly better patient survival in both univariate and multivariate analysis for patients that suffered from diabetes mellitus before transplantation. This has been reported previously [2, 3]; however, reasons for this observation are not exactly clear. An additional, yet unknown, beneficial effect of insulin treatment [2] or a less severe effect on post-transplant survival if diabetes manifests before transplantation compared to new developments after transplantation [3] has been discussed.

In many transplant centres, the use of tacrolimus and mycophenolate mofetil has replaced CsA and azathioprine despite the fact that consistent data favouring one drug over the other in the treatment of paediatric lung transplant recipients are missing [22-25]. In our cohort we found a clear positive impact on survival by the newer immunosuppressive drug CsA and probably also mycophenolate mofetil. The use of these drugs was not evenly distributed over time, with tacrolimus and mycophenolate mofetil being used much more commonly in the later years. As the beneficial effect of tacrolimus was confirmed in multivariate analysis, it seems unlikely that its advantage is only a marker for the better outcome in more recently transplanted patients. However, interaction with other non-assessed parameters, which changed over time, cannot be excluded.

There are no randomised trials to assess the survival benefit of lung transplantation, so the outcome needs to be approached by statistical modelling [26]. In 2008, LIOU and CAHILL [27] presented a study using data from the US CF Foundation Patient Registry and the Organ Procurement and Transplantation Network. The authors applied a proportional hazard model using multiple clinical covariates and the interactions of these covariates with lung transplantation as a time-dependent covariate. They stated that the majority of patients assessed ( 514 children suffering from CF) had an increased risk of death by transplantation, whereas clearly improved survival was shown for only $<1 \%$ of patients [27]. However, several authors have questioned this result and shown clear survival benefit after paediatric lung transplantation [28-30].

The conflicting data on survival benefit of paediatric data adds up to general ethical problems of organ transplantation, such as donor rights, patient allocation, re-transplantation and living donor transplantation. An important point in this aspect is quality of life (QoL) improvement by transplantation, which is an essential parameter besides survival benefit. Unfortunately, data on QoL, especially after lung transplantation in the paediatric age group, is scarce [31-33]. Therefore, conclusions need to be drawn from organ function data. According to the ISHLT registry, the functional status of survivors is very satisfying, with the majority reporting no activity limitations [34]. In our experience, despite the high number of complications, especially in the first months after transplantation, if the patient survives the early post-operative period, QoL generally improves quite clearly. Our patients generally live a rather normal life, go to school and participate in sports as long as their lung function is not significantly impaired by BOS. Psychological problems, such as panic attacks or depression, which are frequently observed in the first weeks after transplantation, generally wane when patients get better; however, this reflects only personal experience and was not systematically assessed in our study.

Another limitation of our study is the small patient number, which decreases the power of statistical analysis. We focused on the accuracy of pre-transplant parameters, only including data within 4 months before transplantation. This leads to missing data in some patients and thus further limits analysis. Low patient number is a general obstacle for the evaluation of paediatric transplantation. According to the ISHLT registry data of 2008, of the 36 centres reporting paediatric transplantations, only five reported more than five procedures per year [5]. Literature on paediatric lung transplantation includes predominantly single centre descriptive studies with relatively few patients. Accordingly, to date, most of the recommendations and the practical handling in the field of paediatric lung transplantation are based on extrapolation of adult lung transplant or other solid organ transplant data, which often is not feasible. Therefore, in the future, multicentre studies are essential to provide information, especially on distinct paediatric aspects of lung transplantation, such as developmental parameters, QoL or pharmacokinetics of applied drugs.

\section{STATEMENT OF INTEREST}

None declared.

\section{REFERENCES}

1 Sweet SC. Pediatric lung transplantation. Proc Am Thorac Soc 2009; 6: 122-127.

2 Hofer M, Benden C, Inci I, et al. True survival benefit of lung transplantation for cystic fibrosis patients: the Zurich experience. J Heart Lung Transplant 2009; 28: 334-339.

3 Liou TG, Adler FR, Cox DR, et al. Lung transplantation and survival in children with cystic fibrosis. N Engl J Med 2007; 357: 2143-2152.

4 Christie JD, Edwards LB, Kucheryavaya AY, et al. The Registry of the International Society for Heart and Lung Transplantation: twenty-seventh official adult lung and heart-lung transplant report - 2010. J Heart Lung Transplant 2010; 29: 1104-1118.

5 Aurora P, Edwards LB, Kucheryavaya AY, et al. The Registry of the International Society for Heart and Lung Transplantation: thirteenth official pediatric lung and heart-lung transplantation report - 2010. J Heart Lung Transplant 2010; 29: 1129-1141.

6 Harmon WE, McDonald RA, Reyes JD, et al. Pediatric transplantation, 1994-2003. Am J Transplant 2005; 5: 887-903.

7 Gorler H, Struber M, Ballmann M, et al. Lung and heart-lung transplantation in children and adolescents: a long-term singlecenter experience. J Heart Lung Transplant 2009; 28: 243-248.

8 Mallory GB, Spray TL. Paediatric lung transplantation. Eur Respir J 2004; 24: 839-845. 
9 Fischer U, Kozyga K, Priebe B, et al. Transplant Jahresbericht 2009: Österreichisches Bundesinstitut für Gesundheitswesen [Transplantation Annual Report 2009]. Vienna, Gesundheit Österreich GmbH, 2010; pp. 55-56.

10 Aigner $\mathrm{C}$, Wisser $\mathrm{W}$, Taghavi S, et al. Institutional experience with extracorporeal membrane oxygenation in lung transplantation. Eur J Cardiothorac Surg 2007; 31: 468-473.

11 Burch M, Aurora P. Current status of paediatric heart, lung, and heart-lung transplantation. Arch Dis Child 2004; 89: 386-389.

12 Huddleston CB, Bloch JB, Sweet SC, et al. Lung transplantation in children. Ann Surg 2002; 236: 270-276.

13 Aurora P, Boucek MM, Christie J, et al. Registry of the International Society for Heart and Lung Transplantation: tenth official pediatric lung and heart/lung transplantation report - 2007. J Heart Lung Transplant 2007; 26: 1223-1228.

14 Elizur A, Sweet SC, Huddleston CB, et al. Pre-transplant mechanical ventilation increases short-term morbidity and mortality in pediatric patients with cystic fibrosis. J Heart Lung Transplant 2007; 26: 127-131.

15 Rithalia A, McDaid C, Suekarran S, et al. A systematic review of presumed consent systems for deceased organ donation. Health Technol Assess 2009; 13: iii, ix-xi, 1-95.

16 Boucek MM, Edwards LB, Keck BM, et al. Registry of the International Society for Heart and Lung Transplantation: eighth official pediatric report - 2005. J Heart Lung Transplant 2005; 24: 968-982.

17 Huddleston CB, Mendeloff EN, Cohen $\mathrm{AH}$, et al. Lung retransplantation in children. Ann Thorac Surg 1998; 66: 199-203.

18 Novick RJ, Stitt LW, Al-Kattan K, et al. Pulmonary retransplantation: predictors of graft function and survival in 230 patients. Pulmonary Retransplant Registry. Ann Thorac Surg 1998; 65: 227-234.

19 Sweet SC. Pediatric lung transplantation: update 2003. Pediatr Clin North Am 2003; 50: 1393-1417.

20 Trulock EP, Christie JD, Edwards LB, et al. Registry of the International Society for Heart and Lung Transplantation: twentyfourth official adult lung and heart-lung transplantation report 2007. J Heart Lung Transplant 2007; 26: 782-795.

21 Aigner C, Jaksch P, Taghavi S, et al. Pulmonary retransplantation: is it worth the effort? A long-term analysis of 46 cases. J Heart Lung Transplant 2008; 27: 60-65.
22 Faro A, Mallory GB, Visner GA, et al. American Society of Transplantation executive summary on pediatric lung transplantation. Am J Transplant 2007; 7: 285-292.

23 Hachem RR, Yusen RD, Chakinala MM, et al. A randomized controlled trial of tacrolimus versus cyclosporine after lung transplantation. J Heart Lung Transplant 2007; 26: 1012-1018.

24 Sollinger HW. Mycophenolate mofetil for the prevention of acute rejection in primary cadaveric renal allograft recipients. U.S. Renal Transplant Mycophenolate Mofetil Study Group. Transplantation 1995; 60: 225-232.

25 Haverich A, Gorler H. Experience with cyclosporine: from revolution to evolution of immunosuppressive protocols in thoracic organ transplantation. Transplant Proc 2004; 36: Suppl. 2, 314S-317S,

26 Kotloff RM. Does lung transplantation confer a survival benefit? Curr Opin Organ Transplant 2009; 14: 499-503.

27 Liou TG, Cahill BC. Pediatric lung transplantation for cystic fibrosis. Transplantation 2008; 86: 636-637.

28 Aurora P, Spencer H, Moreno-Galdo A. Lung transplantation in children with cystic fibrosis: a view from Europe. Am J Respir Crit Care Med 2008; 177: 935-936.

29 Aurora P, Whitehead B, Wade A, et al. Lung transplantation and life extension in children with cystic fibrosis. Lancet 1999; 354: 1591-1593.

30 Egan TM. Solid benefit of lung transplantation for some children with cystic fibrosis. Pediatr Transplant 2008; 12: 125-128.

31 Eskander A, Waddell TK, Faughnan ME, et al. BODE index and quality of life in advanced chronic obstructive pulmonary disease before and after lung transplantation. J Heart Lung Transplant 2011; 30: 1334-1341.

32 Singer LG. Cost-effectiveness and quality of life: benefits of lung transplantation. Respir Care Clin N Am 2004; 10: 449-457.

33 Noll RB, Phipps S. Health-related quality of life after pediatric heart or heart-lung transplantation: where do we go from here? Pediatr Transplant 2005; 9: 134-137.

34 Aurora P, Edwards LB, Christie JD, et al. Registry of the International Society for Heart and Lung Transplantation: twelfth official pediatric lung and heart/lung transplantation report 2009. J Heart Lung Transplant 2009; 28: 1023-1030. 